\title{
Odometry with a Panoramic Radar in Outdoor Environment: use of a Radon-Fourier Transform for the Estimation of the Move without External Sensors
}

\author{
Olivier Fourt, Raphaël Rouveure and Patrice Faure \\ Irstea UR TSCF Technologies et systèmes d'information pour les agrosystèmes \\ 24, avenue des Landais -- BP 50085 -- 63172 Aubière Cedex. http://www.irstea.fr \\ ofourt@hotmail.com,raphael.rouveure@irstea.fr, patrice.faure@irstea.fr
}

\begin{abstract}
In order to make efficient obstacle detection or to create a mapping, SLAM simultaneous localization and mapping being a special case, a good estimation of the sensor's move is needed. Adding dedicated extra sensors to the carrier is the most common solution but not without several limits: sensors have their own accuracy and range of use. Moreover, extra sensors are not often at the same place and so have different moves from one to another. In our outdoor application, the use of a panoramic radar as the main sensor offers another solution: the properties of the Radon's transform and the use of high resolution analysis ESPRIT allows the estimation of the motion's parameters without extra sensors, by the comparisons of successive panoramic maps. The method have been tested in both natural and urban environments and compared to motion estimated by a gyrometer and an odometer.
\end{abstract}

Keywords: Radon Transform, Radar FMCW, ESPRIT, odometry, phase correlation

\section{Introduction}

The design of autonomous or semi-autonomous vehicles is a subject which has been studied for many decades with now several solutions working, even for commercial use. A task which is always unavoidable is the control of the move in the environment, from the simplest obstacle detection or tracking $[37,2,25,12]$ to the creation of a map or the correlation with an existing map, the mapping and map matching $[19,38,26,32,15]$. In robotic applications, the localization in the environment and the mapping could be done either by distinct sensors or either be the results of Simultaneous Localization And Mapping (SLAM) algorithms [31, 28, 8, 14] which allow to step by step build a map and locate the robot from a known starting point [23, 34].

Controlling the move of the carrier is a key point in detection or mapping jobs. Either for choosing a trajectory, building a map by concatenation of a sequence of scans or for a combination of the scans with an external map, the knowledge of the past and possibly future moves of the vehicle is needed. Also a scan on the move may distort the scanning -- an example is the add of a Doppler -- and could complexify [34], thus many applications will chose a strategy of stop and scan. Often the moves' estimations are realized with dedicated extra sensors for several reasons: those sensors are fast, the desired variables are given directly, the data are in a format easy to insert in models such as Kalman filters and lastly a dedicated sensor often has a greater accuracy. Inserting external sensors however has some drawbacks: the first one is the space on the carrier. Often the external sensors are not at the same location as the main scanning 
sensor and thus they have their own move, different from the scanner's one and so requiring extra models and data conversions. The second one is that each sensor has its own condition of use which may differ from one to another and having all sensors working well together is not always easy to achieve. The last one is that each sensor has its own kind of error, being noise trends or else... detecting a default of a sensor and correcting it if possible become an unwanted but necessary task.

The choice of microwave radar as the main scanning senor is offering a good alternative solution for outdoor environment [33]: they have a long range, are resilient to climate, $360^{\circ}$ panoramic view are easy to compute and they remain a class of compact sensors. In our application, the use of a microwave radar as a scanner allows to create a fast sequence of consecutive images between whom the carrier move induce a transform made of a rotation and a translation. Estimating those translation and rotation by the comparison of consecutive images is a problem of map matching. In the field of image processing, the map matching is a subject which has received for many years a great number of answers. So using those works on map matching offers some possibilities to estimate the move of the radar by processing on the radar signal itself without extra external sensors. The goal of this paper is to introduce algorithms combining a robustify map matching processing to our microwave radar who are able to efficiently estimate the move parameters.

The organization of the article is the following: in Section 2 we will present the radar designed in our labs at irstea with its properties. Section 3 will deals with the task of estimating the rotation and translation with the use of a modified Radon-Fourier transform. Section 4 will present the performances of the algorithms, evaluated on several experimental sets.

\section{The Radar for Obstacle Detection and Mapping}

\subsection{The Structure of the Radar}

For short-range distance measurements i.e., few hundreds of meters, the classical pulse radar technology is not the best adapted solution. For example, a precision of 1 meter in distance measurement imposes a precision of $3.3 \mathrm{~ns}$ in time measurement, therefore fast and expensive electronics. We developed the K2Pi microwave radar (meaning $360^{\circ}$ scanning in $\mathrm{K}$ band) using the frequency modulation continuous wave (FMCW) principle. This principle is known and used for several decades [33,6]. When the range is some hundreds of meters, frequency modulation presents the following advantages:

- Low transmission power, as it is the mean power which determines the range, which is safer for the user.

- Transposition of the temporal variables into the frequency domain: a very short delay time $\Delta t$ is transformed into a broad variation of frequency $\Delta f$, easier to measure.

The principle of FMCW radar consists to mix the emitted signal following a linear modulation waveform, with the received signal delayed after reflecting on the targets present within the view of the antenna [27]. Let us consider a sawtooth modulation at frequency $F_{m}$ over a range $\Delta F$ and a distribution of $i$ stationary targets at respective distances $R_{i}$ in the antenna beam. When the echo signal is heterodyned with a portion of the transmitted signal, a beat signal $S_{b}$ will be produced $[33,22]$ : 


$$
S_{b}=k \sum_{i} V_{e} V_{r i} \cos \left(2 \pi\left(2 \Delta F F_{m} \frac{R_{i}}{c}\right) t+\Phi_{t}\right)
$$

$V_{e}$ is the amplitude of the emitted signal, $V_{r i}$ and $\Phi_{\mathrm{i}}$ are respectively the amplitude of received signal and a phase term depending on target $i, c$ the light velocity and $k$ a mixer coefficient. When more than one target is present within the view of the radar, the mixer output will contain a sum $\Sigma F_{b i}$ of frequency components, each of them corresponding to a particular target $i$ :

$$
F_{b i}=2 \Delta F F_{m} \frac{R_{i}}{c}
$$

The range $R_{i}$ to each target may be determined by measuring the individual frequency components by the mean of a Fourier transform for example. At each angle of sight a power spectrum is computed. A panoramic radar image is achieved using a PPI (Plan Position Indicator) representation, which displays range of targets versus angle. Contrary to laser measurement which gives the nearest echo received in the beam, the aperture of the radar beam allows multi-targets detection in the same angle-of-sight. We will notice that moving targets which introduce a Doppler frequency shift are not considered in this paper.

The radar is monostatic, i.e., a common antenna is used for both transmitting and receiving. The rotating antenna achieves a complete $360^{\circ}$ scan around the vehicle in one second, and a radar acquisition is realized each degree. The sensor includes microwave components, electronic devices for emission and reception, data acquisition and signal processing units. A general view of the K2PI radar is shown on the Figure 1. Data acquisition and signal processing units are based on a Pentium M $2 \mathrm{GHz} \mathrm{PC} / 104$ processor card. Computed data are transmitted for visualization and further treatment using an Ethernet link. Main characteristics of the radar are described in the Table 1.

Table 1. Main Characteristics of the K2Pi Radar

\begin{tabular}{lc}
\hline Carrier frequency & $24.125 \mathrm{GHz}$ \\
\hline Bandwidth & $200 \mathrm{MHz}$ \\
\hline EIRP & $18 \mathrm{dBm}$ \\
\hline Range & $3-100 \mathrm{~m}$ \\
\hline Size (length-width-height) & $27-24-30 \mathrm{~cm}$ \\
\hline Weight & $10 \mathrm{~kg}$ \\
\hline
\end{tabular}




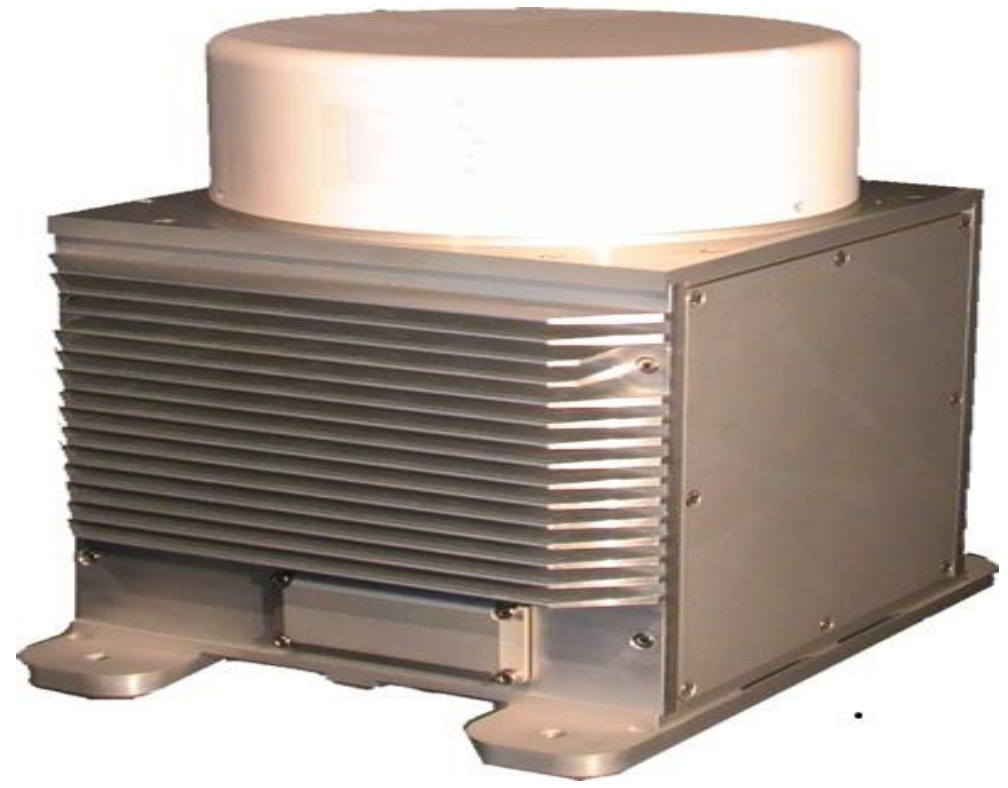

Figure 1. General View of the K2Pi Radar

\subsection{Computation of the Panoramic Maps}

The radar is continuously scanning its environment in all directions, making the acquisition of the returning signal, sum of the echoes from the different possible targets. Datas are grouped by rays corresponding to the return of a frequency ramp of the emitted signal for a given angular position of the antenna. The rotation speed of the antenna and the period of the triangular modulation are synchronized so that the acquisition of a ray corresponds to a rotation of the antenna of 1: a panoramic map is computed on a set of 360 rays, theoricaly equally spaced.

The creations of the panoramic maps are then a two-step process [21, 18, 33]. Firstly the power spectrum of each ray is computed, the result is a map in polar coordinates localization of the targets in the angular and distance (or frequency) plane. Secondly, with the use of the real angular values of the rays, this map is converted to a Cartesian equally spaced grid. This is we called the panoramic radar map, which give the localization of the targets in an $\$ \mathrm{x}-\mathrm{y} \$$ distance plane, the center of the map being the position of the radar, an example is being shown on the Figure 2 which displays a panoramic radar map computed with FFT and the corresponding aerial view.

Usually, spectrums are computed using fast Fourier transforms but for this paper, spectrums are computed using high resolution algorithm. Although several methods like Prony or Pisarenko [13, 16, 29, 3] could be used, the small amount of noise in the signals and the tests have shown that ESPRIT [24] algorithm is the best suited for our work. As a result we have changed from a continuous and noisy spectrum to a discrete and almost noise-free spectrum made of Diracs, the differences could be shown on the Figure 3 which plots the spectrums of a radar ray computed with FFT or ESPRIT. ESPRIT algorithm has been computed using Roland Badeau's DESAM toolbox [4, 3].

Whatever is the method chosen for computed spectrums, the panoramic maps are highly distorted by the move [21,23]: Doppler alters the frequencies estimated but the worse distortion is on the origin point of the rays which change with the move from ray to ray: each radar ray has its own initial point located on the trajectory. The correction of those distortions is a task which requires the knowledge of the move's parameters 
with two possible solutions. The first one is to use some extra sensors to estimate them, this is not always available. The second one is to use algorithms which have a great robustness, enough to work with the distorted datas. As we will show in the following part of this article, it is possible to build algorithms whose goal is to estimate the move's parameters from the distorted datas only.

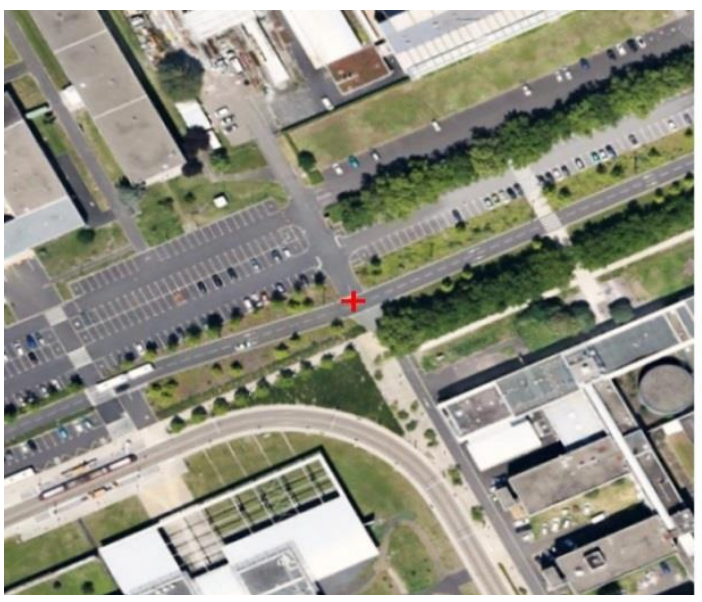

(a)

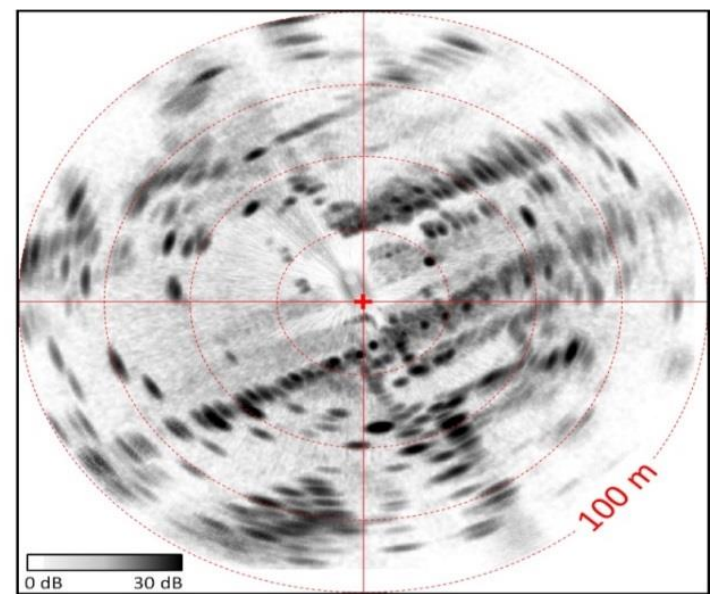

(b)

Figure 2. Example of Radar Image. 2(a) Aerial Image of the Test Zone. The Red Cross Indicates the Radar Position. 2(b) Radar Image Obtained when Spectrum are Computed using Fast Fourier Transforms. The Effect of the Horizontal Antenna Aperture is Clearly Observed for the Largest Distances

\section{Estimation of the Movement Parameters with a Map Matching}

Panoramic maps created by the radar are maps made of pixels: there are a priori no differences between pixels and so point-matching algorithms like Harris [11], Reid's multiple hypothesis tracking [7] or SIFT [39, 17, 1] are of limited use compared to a global algorithm which considers a panoramic map as a single object. Global algorithms also have some interests towards the problems of distortion: when the move is constant, the distortions are similar from maps to maps and there effects will be more on the amplitude of a correlation's peak than on its localization. In order to efficiently estimate the rotation between two images, transformations with a polar structure are of a great interest [20]: their nature often turns a rotation into a translation into the transform domain and thus allowing the estimation of this rotation parameter with a correlation phase algorithm. One of these transformations is the Fourier-Mellin transform [9, 10], a quite old transform which has known a new born with the use of polar-FFT algorithms. For an image $f(r, \theta)$ in polar coordinates, the analytical Fourier-Mellin transform $M_{f \sigma}(k, v)$ is defined by:

$$
M_{f_{\sigma}}(k, v)=\frac{1}{2 \pi} \int_{0}^{+\infty} \int_{0}^{2 \pi} f(r, \theta) r^{\sigma-i v} e^{-i k \theta} d \theta \frac{d r}{r}
$$

with $\sigma>0$, a value of $\sigma=0.5$ is usually recommended. Fourier-Mellin transform is simple and quite fast, even with a coordinate conversion from Cartesian to polar. However the experiments with real datas had proven its accuracy is not enough for our task which has to estimate very small angles and often produce strong errors. As a consequence our tests have leaded us to use another transform with a polar structure: the Radon transform. 


\subsection{Estimation of the Rotation Angle with a Radon Transform}

3.1.1. The Radon Transform and its Properties: In recent years, Hough transform, Trace transform and the related Radon transform have received much attention. In the view of mathematics, Hough transform is a derivative of Radon transform and Radon transform is a special case of Trace transform. These three transforms are able to transform two dimensional images with lines into a domain of possible line parameters, where each line in the image will give a peak positioned at the corresponding line parameters [38, 36, 30]. These have led to many line, circle and curves detection applications within image processing, computer vision. In mutli-resolution analysis, it has also lead to the ridgelet and curvelet transforms. Tomography images are commonly using Radon transform for their reconstruction.

For a two dimensional map $f(x, y)$ defined on a domain $\mathrm{D}$ the $\operatorname{Radon} \operatorname{transform} \mathcal{R}_{f}(D)=$ $\mathcal{R}_{f}(\rho, \theta)$ is the following $[35,5]$ :

$$
\mathcal{R}_{f}(\rho, \theta)=\int_{-\infty}^{+\infty} \int_{-\infty}^{+\infty} f(x, y) \delta(\rho-x \cos \theta-y \sin \theta) d x d y
$$

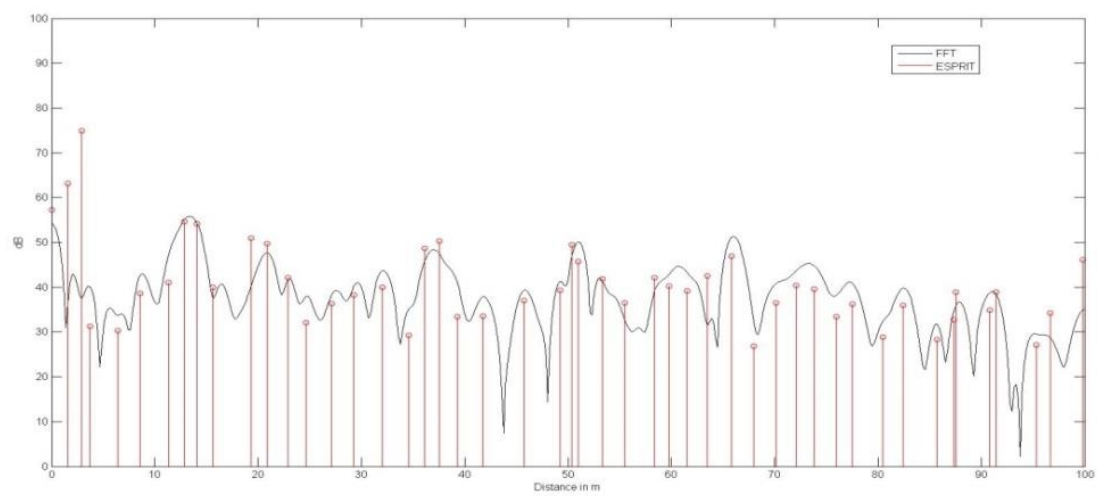

Figure 3. Example of Spectrums Computed with ESPRIT or FFT for a Radar Ray

Radon transform has several properties, a set of the most useful are listed next:

- Linearity

$$
\mathcal{R}_{f+g}(\rho, \theta)=\mathcal{R}_{f}(\rho, \theta)+\mathcal{R}_{g}(\rho, \theta)
$$

- $2 \pi$ periodicity for the angular variable

$$
\mathcal{R}_{f}(\rho, \theta)=\mathcal{R}_{f}(\rho, \theta+2 k \pi) \quad \forall k \in \mathbb{Z}
$$

- Semi-symmetry

- Translation of the map $f(x, y)$ by a vector $(\Delta x, \Delta y)$

$$
\mathcal{R}_{g}(\rho, \theta)=\mathcal{R}_{f}(\rho-\Delta x \cos \theta-\Delta y \sin \theta, \theta)
$$

- Rotation of the map $f(x, y)$ by an angle $\phi$

$$
\mathcal{R}_{g}(\rho, \theta)=\mathcal{R}_{f}(\rho, \theta+\phi)
$$


- Scaling of the map $f(x, y)$ by a factor $\alpha$

$$
\mathcal{R}_{g}(\rho, \theta)=\frac{1}{\alpha} \mathcal{R}_{f}(\alpha \rho, \theta)
$$

3.1.2. Algorithm for the Angle's Estimation: The goal of the process presented in this part is to estimate the rotation between two consecutive panoramic radar maps with the use of the radar datas only. While building and testing the algorithm on real datas, the semi-symmetry property of Radon transform had proved to be troublesome with the estimation of angle with large values: as a consequence, the rotation parameter $\phi$ must be restricted to the interval $[-\pi / 2 ; \pi / 2]$. Between two consecutive panoramic, the move of the radar implies a transform $f \rightarrow g$ made of a rotation of angle $\phi$ and a translation of vector $(\Delta x, \Delta y)$, thus the Radon transform of the two maps are linked by:

$$
\mathcal{R}_{g}(\rho, \theta)=\mathcal{R}_{f}(\rho-\Delta x \cos (\theta+\phi)-\Delta y \sin (\theta+\phi), \theta+\phi)
$$

If we compute $\hat{\mathcal{R}}_{g}(\gamma, \theta)$, result of the one dimensional Fourier transform of $\mathcal{R}_{g}(\rho, \theta)$ on the variable $\rho$ we get:

$$
\widehat{\mathcal{R}}_{g}(\gamma, \theta)=\widehat{\mathcal{R}}_{f}(\gamma, \theta+\phi) \times e^{i 2 \pi \gamma(\Delta x \cos \theta+\Delta y \sin \theta)}
$$

So the magnitude $\left|\widehat{\mathcal{R}}_{g}(\gamma, \theta)\right|$ is a translation of the magnitude $\left|\widehat{\mathcal{R}}_{f}(\gamma, \theta)\right|$ by a factor $\phi$ which is the angle we want to estimate.

Naming $\mathrm{P}_{k} k=1 \ldots n$ the list of the consecutive panoramic radar maps the algorithm for the estimation of the angles $\widehat{\phi_{k}} k=1 \ldots n-1$ between $\mathrm{P}_{k}$ and $\mathrm{P}_{k+1}$ is the following (summarized on the Figure 4):

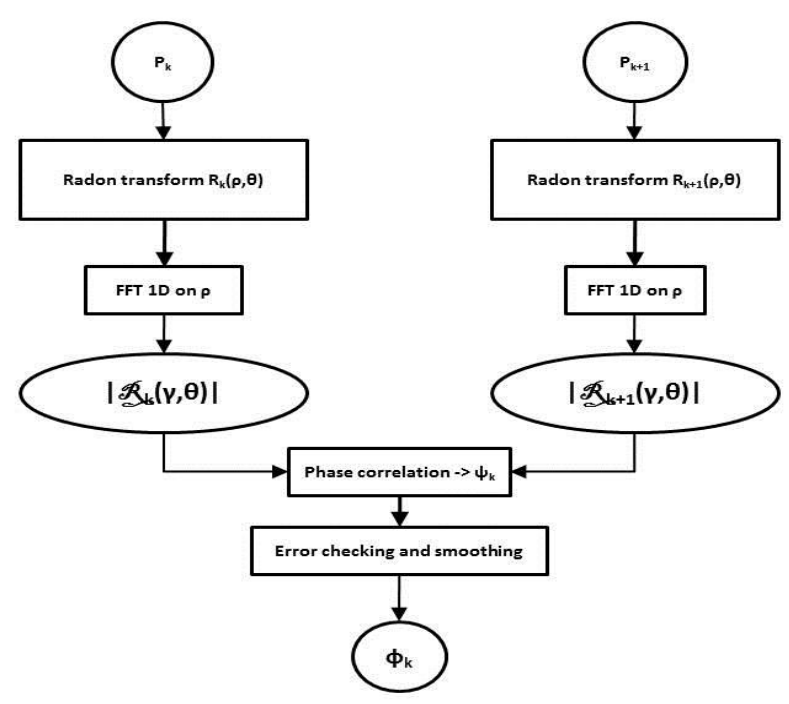

Figure 4. Algorithm for the Estimation of the Angle $\phi_{k}$ between Two Consecutives Panoramic Maps $\mathrm{P}_{k}$ and $\mathrm{P}_{k+1}$ with a Radon Transform 
1. $\forall k \in\{1 \ldots n\}$ compute $\mathcal{R}_{\mathrm{P}_{k}}(\rho, \theta)$, Radon transform of $\mathrm{P}_{k}$. The transforms are computed for a set of angles $\theta_{j}$ equally spaced on the interval $[0 ; \pi]$.

2. $\forall k \in\{1 \ldots n\}$ apply a $1 \mathrm{D}$ Fourier transform to $\mathcal{R}_{\mathrm{P}_{k}}(\rho, \theta)$, to obtain $\widehat{\mathcal{R}}_{\mathrm{P}_{k}}(\gamma, \theta)$.

3. $\forall k \in\{1 \ldots n-1\}$ apply a phase correlation algorithm to $\left|\widehat{\mathcal{R}}_{\mathrm{P}_{k}}(\gamma, \theta)\right|$ and $\left|\widehat{\mathcal{R}}_{\mathrm{P}_{k+1}}(\gamma, \theta)\right|$ to obtain the angles $\psi_{k}$

4. Apply a post-processing algorithm to $\psi_{k} k=1 \ldots n-1$ for error removal and smoothing to obtain $\widehat{\phi_{k}} k=1 \ldots n-1$ the list of rotation angles.

The Radon transform is computed on a set of angles $\theta_{j}$ equally spaced on the interval $[0 ; \pi]$. The estimation of the angles at step 3 may lead to failure and thus strong errors: check and correct those errors is the goal of the post-processing algorithm at step 4. It also enables and increases of the accuracy by computing predictions of the estimated angles or inserting datas form an external sensor. This post-processing algorithm is:

1. Compute $\tilde{\psi}_{k} k=1 \ldots n-1$ by applying a median filter of order 5 to $\psi_{k} k=1 \ldots n-1$.

2. $\forall k \in\{1 \ldots n-1\}$ if $\left|\tilde{\psi}_{k}-\psi_{k}\right| \geq 0.05$ then $\tilde{\phi}_{k}=\frac{\widetilde{\psi}_{k-2}+\widetilde{\psi}_{k-1}+\widetilde{\psi}_{k+1}+\widetilde{\psi}_{k+2}}{4}$ else $\widetilde{\phi}_{k}=\psi_{k}$

3. Improve accuracy: compute predictors by fitting polynomials on the sets $\left\{\tilde{\phi}_{k-2} ; \tilde{\phi}_{k-1} ; \tilde{\phi}_{k+1} ; \tilde{\phi}_{k+2}\right\}$, compares the results with $\tilde{\phi}_{k}$ and possible external datas to get $\widehat{\phi_{k}} k=1 \ldots n-1$.

\subsection{Estimation of the Translations Parameters}

3.2.1. Model for the Radar's Move: In the experiments, the radar is installed on a carrier, usually a car. Its move is modeled at the simplest as the displacement of a vector from a point $M_{k}$ to a point $M_{k+1}$ with a trajectory locally assimilated to a circle. Thus with this model the translation between the two points $M_{k}$ and $M_{k+1}$, the change of course, a rotation of angle $\phi_{k}$ and the displacement $D_{k}$ are linked together by the following equations:

$$
\begin{gathered}
\Delta_{x}=D_{k} \cos \left(\psi_{k}+\frac{\phi_{k}}{2}\right) \\
\Delta_{y}=D_{k} \sin \left(\psi_{k}+\frac{\phi_{k}}{2}\right) \\
\psi_{k+1}=\psi_{k}+\phi_{k}
\end{gathered}
$$

This model is quite simple, it is however enough for our jobs as long as there are no sliding or spinning effects that may decorrelate the translation and the rotation parts of the moves.

For estimating of the translation between two maps that differ by a translation and a rotation, most algorithms are firstly estimating the rotation then rotating back one of the maps in order to have two maps differing only by a translation and finally estimating the translation parameters. This strategy still holds true for our system however the move of the radar has some consequences on the translation parameters and so the accuracy of their estimation. The lateral move and the longitudinal move usually are not in the same range of values, often by a factor between 10 and 100, thus a set of analysis 
parameter suited for the estimation of the first one will not efficiently estimate the second one and reciprocally. However according to the model used, the two translation parameters are linked together with the rotation parameter. Naming $\Delta_{x}$ the longitudinal move and $\Delta_{y}$ the lateral move we have the relationship:

$$
\Delta_{y}=\Delta_{x} \tan \left(\frac{\phi}{2}\right)
$$

3.2.2. Algorithm: The algorithm for the estimation $\widehat{\Delta}_{x}(k) k=1 \ldots n-1$ and $\widehat{\Delta}_{y}(k) k=$ $1 \ldots n-1$ of the translations parameters between consecutive panoramic radar maps $\mathrm{P}_{k} k=1 \ldots n$ with the rotation parameter $\phi_{k} k=1 \ldots n-1$ known is the following (summarized on the Figure 5):

1. $\forall k \in\{1 \ldots n-1\}$ get the panoramic maps $\mathrm{P}_{k}$ and $\mathrm{P}_{k+1}$, then apply an inverse rotation of $\phi_{k}$ to $\mathrm{P}_{k+1}$, in order to get $M_{k+1}$.

2. $\forall k \in\{1 \ldots n-1\}$ estimate the translations between $\mathrm{P}_{k}$ and $M_{k+1}$ with a phase correlation algorithm: $\widetilde{\Delta_{x}}(k)$ and $\widetilde{\Delta_{y}}(k)$.

3. Apply a median filter of order 5 to $\widetilde{\Delta_{x}}(k)$, compares the results with a moving average filter in order to remove errors and get $\widehat{\Delta}_{x}(k) k=1 \ldots n-1$

4. $\forall k \in\{1 \ldots n-1\}$ compute $\widehat{\Delta}_{y}(k)=\widehat{\Delta}_{x}(k) \tan \left(\frac{\phi_{k}}{2}\right)$

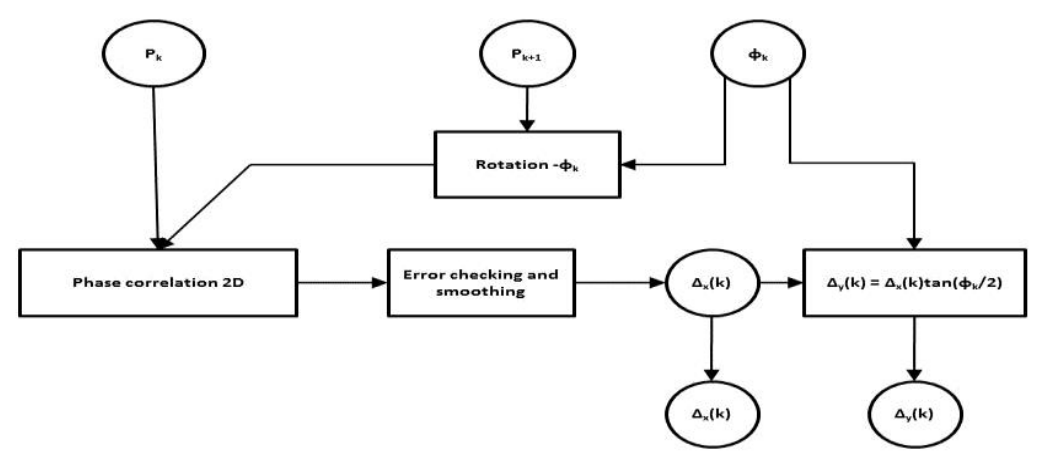

Figure 5. Algorithm for the Estimation of Translations $\Delta_{x}(\boldsymbol{k})$ and $\Delta_{y}(\boldsymbol{k})$ between Two Consecutives Panoramic Maps $\mathbf{P}_{k}$ and $\mathbf{P}_{k+1}$ with the Rotation $\phi_{k}$ between them and Phase Correlation

The fast estimation $\widetilde{\Delta_{y}}(k) k=1 \ldots n-1$ could be enough for long and almost rectilinear trajectories. When the model of the carrier's move no longer holds true, it is often due to sliding or spinning effects and the translation may become decorrelate from the rotation. The equation 7 could no longer be used, however in those situations, $\Delta_{x}$ and $\Delta_{y}$ are in the same range of value and $\widehat{\Delta}_{y}(k)$ is computed by filtering $\widehat{\Delta}_{y}(k)$ in the same way as for $\hat{\Delta}_{x}(k)$. 


\section{Tests of the Algorithms}

Experiments were conducted in several environments, mostly urban but also in nature. Four data sets have been used: the first one is in a natural environment (exp1), the second one is a rectilinear and slow speed move on one of the university's parking $(\exp 2)$ and the third and fourth one are records in urban area respectively denominated exp3 and exp4, exp3 being records in a residential area and exp4 in a semi-industrial area. Exp1 data is a set of 98 maps, exp2 a set of 275 maps, exp3 a set of 478 maps and exp4 a set of 451 maps.

The results of the experiments are given in term of absolute error on the estimation of $\phi$ (in radian), $\Delta_{x}$ and $\Delta_{y}$ (in meter) and not relative error in order to avoid divisions by zero. The rotation angle reference is given by an external gyrometer sensor and the translations references are given by an external odometer. The tests have shown that the errors estimations have a fat tail: the kurtosis parameter is always greater than 5 . In a first approximation the errors seemed to be symmetric, unfortunately we don't have the private track for a long duration (several hours) tests needed to improve those assessments. The existence of some strong errors requires the use of robust statistics in order to qualify the properties of the error: the robust mean computed by excluding the extremes values and the interquartile range, a robust estimator of the dispersion. For a random variable $V$ with probability law $P(V)$, one defines:

$$
\begin{array}{r}
Q_{1}=v / P(V \leq v) \geq 0.25 \text { and } P(V \geq v) \geq 0.75 \text { first quartile } \\
Q_{3}=v / P(V \leq v) \geq 0.75 \text { and } P(V \geq v) \geq 0.25 \text { third quartile } \\
i q r=\left|Q_{3}-Q_{1}\right| \text { interquartile range }
\end{array}
$$

\subsection{Estimation of the Rotation}

The Table 2 lists the results of the experiments in term of statistics of the error in radian with $m$ being the mean of the error, M being the median of the error and iqr the interquartile range of the error. Exp2 experiment has far greater results because it is a move with almost no rotation. Poorer results of exp4 may imply it's trajectory on an edge of a hill which is distorting the radar's returning signals. On the Figure 3 we are presenting the angles estimated by a gyrometer and by our algorithm for the experiment exp3, Figure 3 being a zoom on the curves for the maps numbered between 100 and 270. We could see that although our algorithm is quite efficient, it still has trouble with strong angle changes, especially when immediately followed by the opposite change. It may also swap some tiny change but this is not always a drawback: it will smooth the irregularities in car driving. 


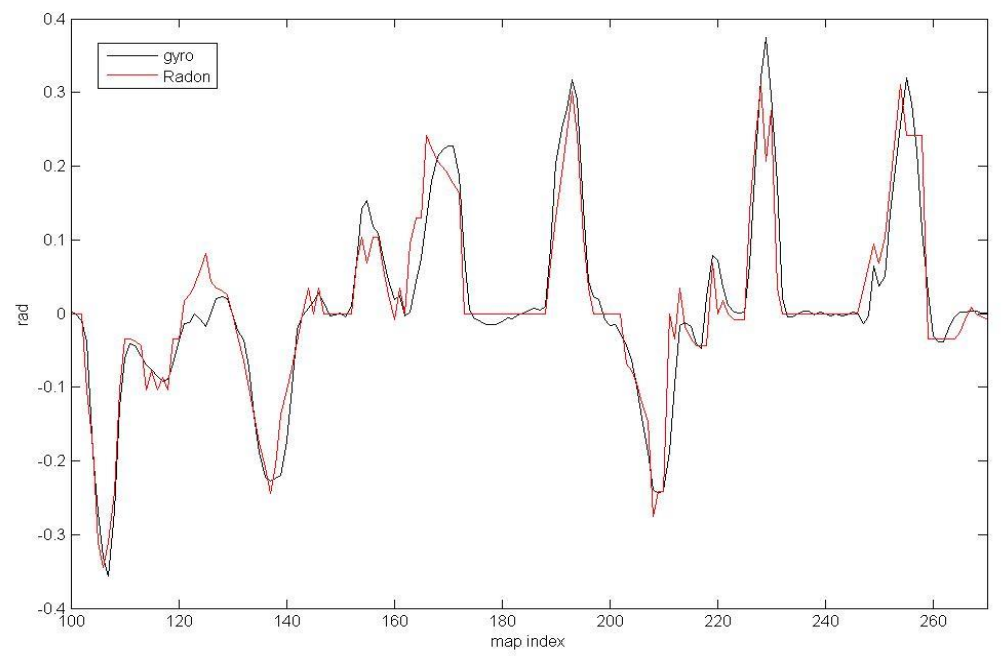

Figure 6. Angles Estimated by Gyrometer and Radon Transform for exp3, for Maps $n 100$ to 270 of a Set of 478 Radar Maps

Table 2. Error's statistics in radian on the estimation of the angles for the four experiments

\begin{tabular}{|c|c|c|c|c|}
\hline experiment & $\exp 1$ & $\exp 2$ & $\exp 3$ & $\exp 4$ \\
\hline $\mathrm{m}$ & $3.51 \times 10^{-4}$ & $1.83 \times 10^{-4}$ & $-2.5 \times 10^{-3}$ & $6.11 \times 10^{-4}$ \\
\hline $\mathrm{M}$ & $-5.17 \times 10^{-6}$ & $-1.39 \times 10^{-5}$ & $-4.52 \times 10^{-6}$ & $-4.19 \times 10^{-5}$ \\
\hline$i q r$ & 0.024 & $1.16 \times 10^{-4}$ & 0.026 & 0.039 \\
\hline
\end{tabular}

Table 3. Error's Statistics in Meter on the Estimation of the Longitudinal Move $\Delta_{x}$ for the Four Experiments

\begin{tabular}{|c|c|c|c|c|}
\hline experiment & $\exp 1$ & $\exp 2$ & $\exp 3$ & $\exp 4$ \\
\hline $\mathrm{m}$ & 0.07 & 0.0031 & -0.09 & -0.086 \\
\hline $\mathrm{M}$ & 0.08 & 0 & -0.06 & -0.035 \\
\hline$i q r$ & 0.23 & $? ?$ & 0.24 & 0.29 \\
\hline
\end{tabular}




\subsection{Estimation of the Translations}

As for the estimation of the rotation, the Table 3 and Table 4 list the results of the experiments in term of statistics of the absolute error in meters. Exp2 experiment has far greater results because it is a move with almost no rotation and a slow speed always below $3 \mathrm{~m} / \mathrm{s}$. The results on the estimation of the lateral translation parameter $\widehat{\Delta}_{y}(k) k=$ $1 \ldots n-1$ are showing the usefulness of a good rotation estimation: the rotation parameter seems to smooth the effect of a bad estimation of $\hat{\Delta}_{x}(k) k=1 \ldots n-1$. The interquartile ranges for exp2 experiment are undefined because they are below numerical precision. Percentile could be computed for values of $15 \%$ and $84 \%$ giving a range between them of $4.4 \times 10^{-3}$ on $\Delta_{x}$ error estimation and a range of $1.9 \times 10^{-6}$ on $\Delta_{y}$ error estimation. On the Figure 7 and Figure 8 we are presenting the $\Delta_{x}$ and $\Delta_{y}$ moves estimated by an odometer and our algorithm for exp3 experiment, figures being a zoom on the curves for the maps numbered between 298 and 435 . We could see that $\Delta_{y}$ estimations are always quite good but for $\Delta_{x}$ estimation there are sometimes some errors in exp3 experiment. A look at the datas shows that this may happen with strong variations on the moves and / or the rotation parameter which means a fast move of the radar's carrier. Exp1 and exp4 experiments have the same kind of results opposed to the accuracy of exp2 experiment with its slow move.

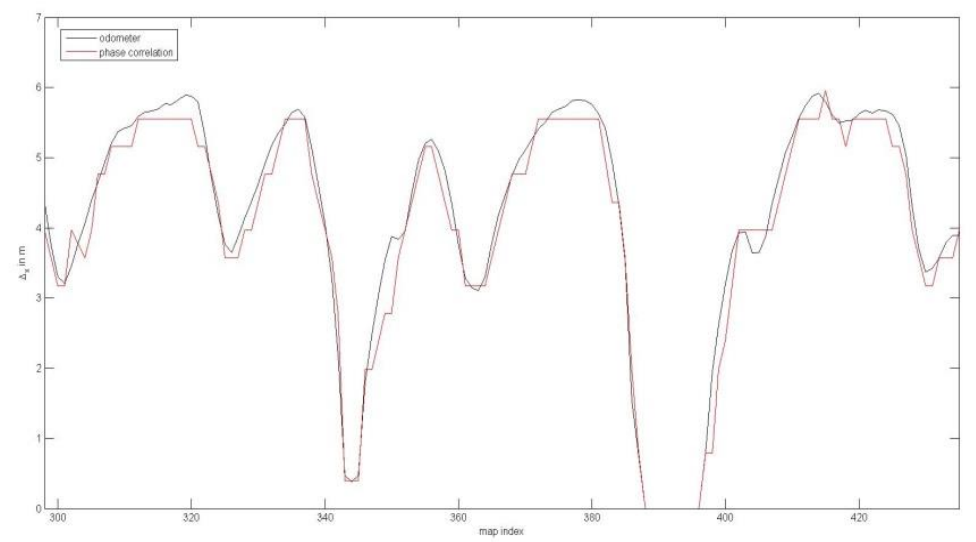

Figure 7. Longitudinal Move $\Delta_{x}$ Estimated by Odometer and fft Phase Correlation for exp3 Experiment, for Maps $n^{\circ} 298$ to 435 of a Set of 478 Radar Maps

Table 4. Error's Statistics in Meter on the Estimation of the Longitudinal Move $\Delta_{y}$ for the Four Experiments

\begin{tabular}{|c|c|c|c|c|}
\hline experiment & $\exp 1$ & $\exp 2$ & $\exp 3$ & $\exp 4$ \\
\hline $\mathrm{m}$ & $4.6 \times 10^{-3}$ & $-1.47 \times 10^{-6}$ & $-3.1 \times 10^{-3}$ & $-3.2 \times 10^{-3}$ \\
\hline $\mathrm{M}$ & $2.45 \times 10^{-4}$ & 0 & 0 & 0 \\
\hline $\mathrm{iqr}$ & $7.4 \times 10^{-3}$ & $? ?$ & $1.9 \times 10^{-3}$ & $3.8 \times 10^{-3}$ \\
\hline
\end{tabular}




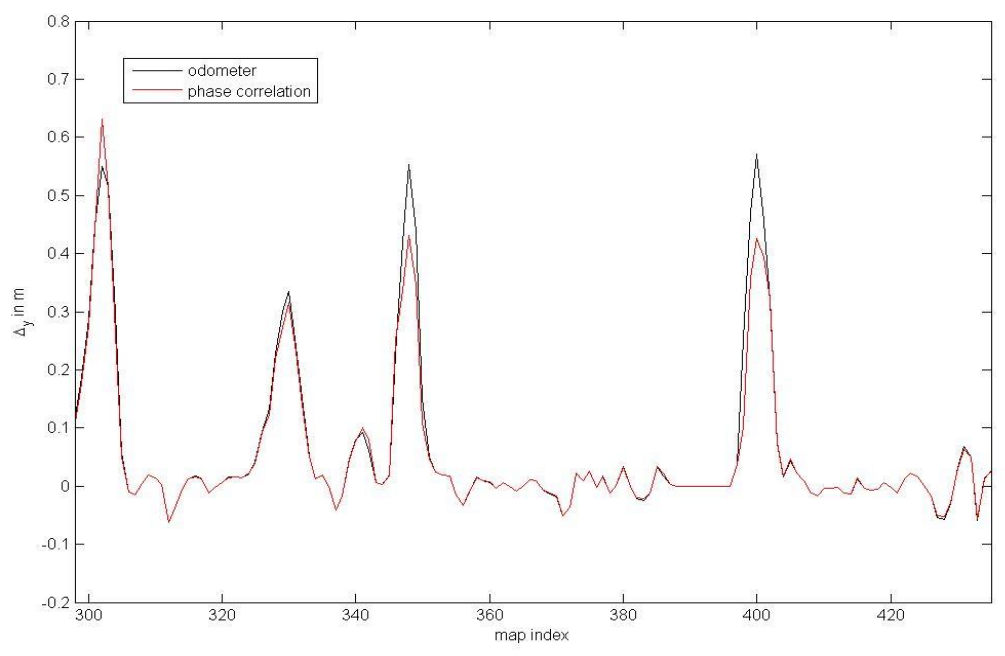

\section{Figure 8. Lateral Move $\Delta_{y}$ Estimated by Odometer and fft Phase Correlation for exp3 Experiment, for Maps $n^{\circ} 298$ to 435 of a Set of 478 Radar Maps}

\section{Conclusion}

In this paper, we have shown how map matching algorithms based on Radon transform could be applied on the signal of a continuously modulated frequency radar used in panoramic mode in order to efficiently estimate the displacements of the radar without using extra external sensor. The experiments have also shown the upper bounds in using our algorithms: a maximum value to the speed, around $3 \mathrm{~m} / \mathrm{s}$ and a maximum value to the rotation, the first one being the most restrictive factor. The improvement of the results will rely on the next generation of sensor which will be a radar with a greater sampling rate and possibility of a greater rotation speed of the antenna. This will allow scanning panoramic map at a greater speed than today and for the odometry, it will allow a greater upper bound on the speed. Presently, our algorithms have several possible uses: firstly they could be another source of knowledge in a Kalman filter used to estimate the move of a robot, a task commonly encountered in SLAM applications. Secondly, when the radar is already used together with a gyrometer an odometer or a GPS, our methods offer a safety mode allowing the estimation of the move with a possible loss of accuracy when other sensors are disabled. Lastly, in some application it may be difficult to use external sensor: water environment makes hard the use of an odometer, canyon and forest cover could shut down the GPS. In those jobs like the mapping of a river from the boat, the estimation of the move from the radar's signals is the only remaining possibility.

\section{Acknowledgements}

This work is part of PELICAN project $\mathrm{N}^{\circ}$ ANR-2010-EMMA-033 supported by the Agence Nationale de la Recherche, and part of QUAD-AV project from ICT-Agri European program. 


\section{References}

[1] F. Alhwarin, D. Ristic-Durrant and A. Gräser. Vf-sift: Very fast sift feature matching. In DAGMSymposium, pages 222-231, (2010).

[2] A. Ayoun and P. Smets. Data association in multi-target detection using the transferable belief model. International Journal of Intelligent Systems. 16:1167-1182, (2000).

[3] Roland Badeau. High resolution methods for estimating and tracking modulated sinusoids. Application to music signals. PhD thesis, Ecole Nationale Supérieure des Télécommunications, (2005).

[4] Roland Badeau and Bertrand David. Desam toolbox (2010).

[5] Yufang Cai, Kuan Shen and Jue Wang. Application of radon transform in ct image matching.

[6] G. Collette and R. Labrousse. Un altimètre radioélectrique à modulation de fréquence. l'Onde Electrique. 37(363), (1957).

[7] Ingemar J. Cox and Sunita L. Hingorani. An efficient implementation of reid's multiple hypothesis tracking. IEEE Trans. Pattern Anal. Mach. Intell. 18(2):138-150, (1996).

[8] M. W. M. Gamini Dissanayake, Paul Newman, Steven Clark, Hugh F. Durrant-whyte and M. Csorba. A solution to the simultaneous localization and map building (slam). IEEE Transactions on Robotics and Automation. 17:229-241, (2001).

[9] Xiaoxin Guo, Zhiwen Xu, Yinan Lu, Zhanhui Liu, and Yunjie Pang. Image registration based on pseudopolar fft and analytical fourier-mellin transform. In ICIC (1), pages 30-39, (2005).

[10] Xiaoxin Guo, Zhiwen Xu, Yinan Lu, and Yunjie Pang. An application of fourier-mellin transform in image registration. In CIT, pages 619-623, (2005).

[11] Chris Harris and Mike Stephens. A combined corner and edge detector. In In Proc. of Fourth Alvey Vision Conference, pages 147-151, (1988).

[12] Anil K. Jain, Robert P. W. Duin and Jianchang Mao. Statistical pattern recognition: A review. IEEE TRANSACTIONS ON PATTERN ANALYSIS AND MACHINE INTELLIGENCE. 22(1):4-37, (2000).

[13] Julius Kusuma. Parametric frequency estimation: Esprit and music. (2002).

[14] John J. Leonard, Hans Jacob and S. Feder. A computationally efficient method for large-scale concurrent mapping and localization. In Proceedings of the Ninth International Symposium on Robotics Research, pages 169-176. Springer-Verlag, (1999).

[15] David G. Lowe. Distinctive image features from scale-invariant keypoints. International Journal of Computer Vision. 60(2):91-110, (2004).

[16] M. S Mackisack, M. R Osborne, and G. K Smyth. A modified prony algorithm for estimating sinusoidal frequencies. Journal of Statistical Computation and Simulation. 49(1-2):111-124, (1994).

[17] Dominic R. Maestas, Ron Lumia, Gregory P. Starr and John E. Wood. Scale invariant feature transform (sift) parametric optimization using taguchi design of experiments. In ICIRA (1), pages 630-641, (2010).

[18] Marie-Odile Monod, Roland Chapuis, Philippe Gosset, Raphaël Rouveure, Damien Vivet, Franck Gérossier, Patrice Faure, Paul Checchin, Laure Moiroux, Pierre Guérin, Thierry Humbert and Joël Morillon. Radar panoramique hyperfréquence pour la localisation et la cartographie simultanées en environnement extérieur. Traitement du Signal. (2010).

[19] B.Srinivasa Reddy and B.N. Chatterji. correspondence an fft-based technique for translation, rotation, and scale-invariant image registration.

[20] B.S. Reddy and B.N. Chatterji. An fft-based technique for translation, rotation, and scale-invariant image registration. Image Processing, IEEE Transactions on. 5(8):1266-1271, aug, (1996).

[21] R. Rouveure, P. Faure and M.O. Monod. Radar imager for perception and mapping in outdoor environment. In Advanced Concepts for Intelligent Vision Systems, ACIVS 2009, 28/09/2009-02/10/2009, Bordeaux, FRA / Actes du colloque ACIVS 2009, page 12. Springer-Verlag, (2009).

[22] R. Rouveure, M.O. Monod and P. Faure. Multiple targets detection with a fmcw radar dedicated to mobile robotics. In RADAR 2004 International Conference on Radar Systems, Toulouse. 18-22 octobre 2004, page 3, (2004).

[23] R. Rouveure, M.O. Monod and P. Faure. High resolution mapping of the environment with a ground-based radar imager. In IEEE International Conference on Radar Systems (Radar 2009), Bordeaux, France. (2009).

[24] R. Roy and T. Kailath. Esprit - estimation of signal parameters via rotational invariance techniques. IEEE Trans. Acoustics, Speech, Signal Processing. 37 (7):984-995, July (1989).

[25] David Serby and Luc Van Gool. Probabilistic object tracking using multiple features. In IEEE International Conference of Pattern Recognition (ICPR). pages 184-187, (2004).

[26] Khurram Shafique and Mubarak Shah. A non-iterative greedy algorithm for multi-frame point correspondence. In IEEE Transactions on Pattern Analysis and Machine Intelligence. pages 51-65, (2003).

[27] M.I. Skolnik. Introduction to radar systems. Electrical Engineering Series, McGraw-Hill International Editions, (1980).

[28] Dorian J. Spero and Ray A. Jarvis. A review of robotic slam, (2007). 
[29] G. W. Stewart. An updating algorithm for subspace tracking. IEEE Trans. Signal Processing, 40:1535-1541, (1992).

[30] Imants D. Svalbe. Exact, scaled image rotation using the finite radon transform. In DGCI, pages 446-456, (2009).

[31] Sebastian Thrun. Robotic mapping: A survey. In Exploring Artificial Intelligence in the New Millenium. Morgan Kaufmann, (2002).

[32] C. J. Veenman, M. J. T. Reinders and E. Backer. Resolving motion correspondence for densely moving points. IEEE Transactions on Pattern Analysis and Machine Intelligence, 23:54-72, (2001).

[33] Marie-Odile Villard-Monod. Radar à Modulation de Fréquence: un nouveau capteur pour l'environnement et la robotique mobile d'extérieur. PhD thesis, Université Paris VI, (1995).

[34] Damien Vivet. Perception de l'environnement par radar hyperfréquence. Application à la localisation et la cartographie simultanées, à la détection et au suivi d'objets mobiles en milieu extérieur. PhD thesis, , Université Blaise Pascal - Clermont II, (2011).

[35] Xuan Wang, Bin Xiao, Jian-Feng Ma and Xiu-Li Bi. Scaling and rotation invariant analysis approach to object recognition based on radon and fourier-mellin transforms. Pattern Recognition, 40(12):3503 - 3508, (2007).

[36] Bin Xiao, Jian-Feng Ma, and Jiangtao Cui. Combined blur, translation, scale and rotation invariant image recognition by radon and pseudo-fourier-mellin transforms. Pattern Recognition, 45(1):314-321, (2012).

[37] Alper Yilmaz, Omar Javed and Mubarak Shah. Object tracking: A survey, (2006).

[38] Jiangsheng You, Weiguo Lu, Jian Li, Gene Gindi and Zhengrong Liang. Image matching for translation rotation and uniform scaling by the radon transform. In ICIP (1)'98, pages 847-851, (1998).

[39] Jie Zhao, Li-Juan Xue and Guo-Zun Men. Optimization matching algorithm based on improved harris and sift. In ICMLC, pages 258-261, (2010).

\section{Authors}

Olivier Fourt, was born in 1979. He received an engineering degree from the Institut National Polytechnique de Grenoble in 2001 and the PHD degree from the Université ParisSud (Orsay) in 2008 in Signal Processing. Works in research and engineering in the domains of signal processing. Mains interests are in sparse analysis, fast algorithms and robust algorithms.

Patrice Faure, was born in 1958. He received the D.E.A degree in Microelectronics from University of Montpellier, France in 1984. He is associated with the Ecotechnologies department at the National Research Institute of Science and Technology for Environment and Agriculture (Irstea), France. His research interests include the development of new sensors for environmental application. His work deals with signal processing and technology in microwaves and electronics domain.

Raphaël Rouveure, was born in 1966. He received the electrical engineering degree from the Polytech'Clermont School of Engineering, Clermont-Ferrand, France in 1990 and the $\mathrm{Ph} . \mathrm{D}$ degree in Sciences For Engineer from the University of Clermont 2, France in 2001. He is associated with the Ecotechnologies department at the National Research Institute of Science and Technology for Environment and Agriculture (Irstea), France. His current research activities are in the development of new sensors for environmental application. His work deals with signal processing and technology in microwaves domain. 
International Journal of Signal Processing, Image Processing and Pattern Recognition Vol.7, No.2 (2014) 